\title{
Plasmonic nanostructures made from aluminum fabricated by EUV interference lithography
}

\author{
Y. Ekinci ${ }^{\mathrm{a}}$, H. H. Solak ${ }^{\mathrm{b}}$, C. David ${ }^{\mathrm{b}}$, and J. F. Löffler ${ }^{\mathrm{a}}$ \\ ${ }^{a}$ Laboratory of Metal Physics and Technology, Department of Materials, ETH Zurich, Wolfgang- \\ Pauli-Str. 10, 8093 Zurich, Switzerland \\ E-mail: yasin.ekinci@mat.ethz.ch \\ b Laboratory for Micro- and Nanotechnology, Paul Scherrer Institute, 5232 Villigen-PSI, \\ Switzerland
}

\begin{abstract}
Extreme Ultra Violet (EUV) interference lithography was employed for fabrication of large-area, sub-100 nm scale $\mathrm{Al}$ nanoparticles as well as free-standing aluminum hole arrays. We studied the optical properties of the fabricated $\mathrm{Al}$ nanostructures. The marked difference in $\mathrm{Al}$, compared to $\mathrm{Au}$ and $\mathrm{Ag}$, is that its plasmon resonances lie in the UV range. Thus, the high-frequency plasmon resonance of $\mathrm{Al}$ nanostructures enables the extending of plasmonic research into the UV range and opens up new possible applications of plasmonics.
\end{abstract}

Keywords: spectroscopy, metal nanoparticles, surface plasmons

\section{INTRODUCTION}

The emerging research field of plasmonics deals with collective electronic excitations confined to conducting surfaces (surface plasmon polaritons) or nanoparticles and nanostructures (localized surface plasmons). Exploitation of surface plasmons promises many interesting applications in nanophotonics, data storage, non-linear optics, surface-enhanced Raman spectroscopy, fluorescence spectroscopy, and biochemical sensors ${ }^{1-3}$. Owing to their optical properties, Ag and $\mathrm{Au}$ are commonly used in plasmonic devices working in the visible and near-infrared ranges. At short wavelengths these materials absorb strongly due to the interband transitions which set on below the wavelengths of about $590 \mathrm{~nm}$ and 350 $\mathrm{nm}$ for $\mathrm{Au}$ and $\mathrm{Ag}$, respectively ${ }^{4}$. On the other hand, $\mathrm{Al}$ exhibits negligible interband transitions down to a wavelength of $200 \mathrm{~nm}$ due to its free-electron-like character and has a high bulk-plasmon frequency $\left(\omega_{p, b u l k}=15 \mathrm{eV}\right)^{5}$. For these reasons, plasmon resonances of $\mathrm{Al}$ nanostructures generally lie in the UV range. By experimenting with this material, which has a plasmon frequency that is vastly different from the commonly employed Au and Ag systems, one can gain further insight into plasmonic phenomena. Moreover, development of plasmonic devices in the UV range may lead to new applications such as UV bandpass filters. In addition, operating at short wavelengths can increase the efficiency and sensitivity and reduce the size of plasmonic devices.

In this paper we discuss the plasmonic characteristics of Al nanostructures. In Sec. 2, we describe our fabrication method for these structures. In Sec. 3, we discuss plasmon resonances of Al nanoparticles on dielectric substrates. In Sec. 4, we present one specific manifestation of plasmon resonances of Al nanostructures, namely extraordinary optical 
transmission through subwavelength Al hole arrays. Finally, in Sec. 5 we present our main conclusions and the possible applications of Al plasmonic devices.

\section{EUV INTERFERENCE LITHOGRAPHY}

In plasmonic research, e-beam lithography and focused ion beam (FIB) lithography are the preferred methods for fabricating high-resolution metallic nanostructures of arbitrary shapes. Periodic nanostructures, in general, are fabricated over small areas and characterized with a micro-spectrometer. For Al nanostructures, characterization of the optical response on small-area nanostructures requires UV compatible focusing optics, which is usually not available in standard equipments. Standard spectrometry equipment without any focusing optics can be used for characterization of large area samples but the fabrication process would take very long with serial lithography tools such as e-beam or FIB. In order to overcome these problems faced, we used a new fabrication approach in which we employed extreme-ultraviolet interference lithography (EUV-IL), physical deposition of metal, and lift-off.

The EUV exposures were performed at the XIL beamline of the Swiss Light Source ${ }^{6}$. The interference setup uses transmission gratings and coherent EUV light of $13.4 \mathrm{~nm}$ wavelength as shown in Fig. 1(a). The diffractive metal gratings are patterned on thin silicon nitride membranes using e-beam lithography. This method creates a highresolution and high-contrast aerial image which enables us to print dense lines with resolution down to $12.5 \mathrm{~nm}$ halfpitch $^{7}$. Periodic line/space patterns are obtained by two-beam interference, while four-beam interference provides hole array and dot array patterns. Fig. 1(b) shows a scanning electron microscopy (SEM) image of a dot array patterned by EUV-IL. This high resolution potential combined with high throughput of producing nanostructures over large areas with short exposure times makes EUV-IL a powerful tool for the fabrication of plasmonic structures.
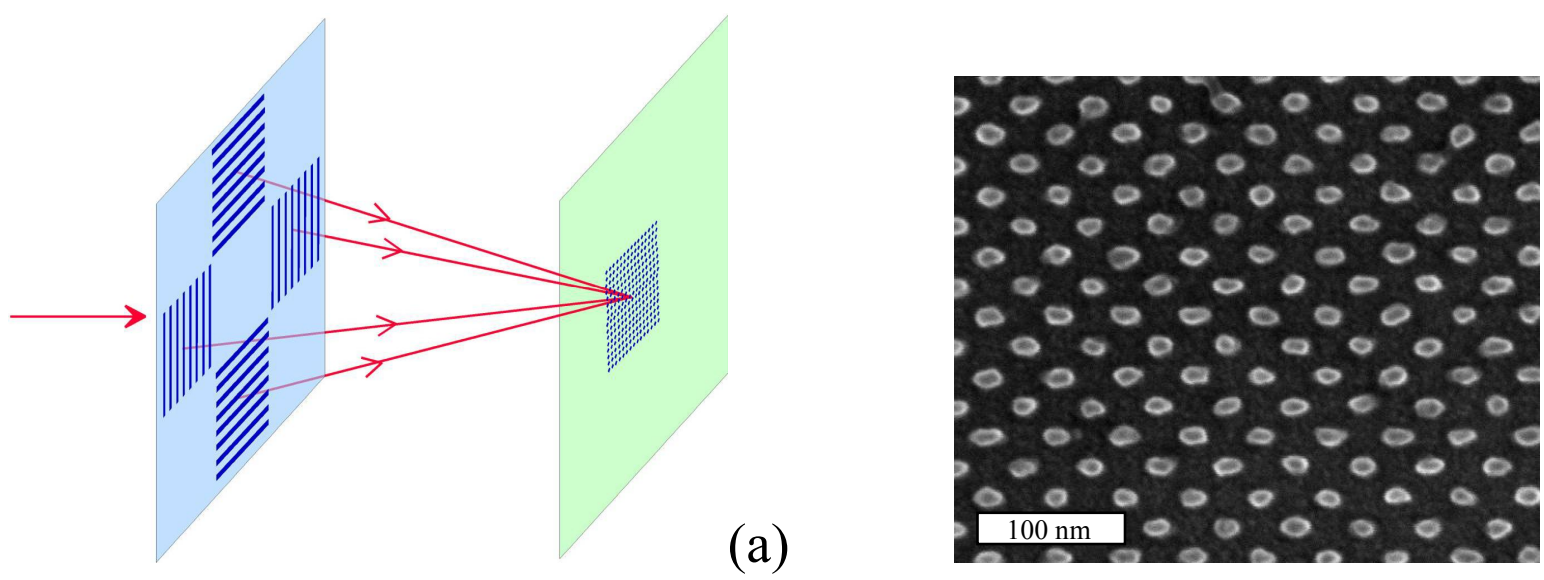

(b)

Figure 1: (a) Sketch of EUV four-beam interference setup. The interference pattern is created by four transmission gratings and coherent light of $13.4 \mathrm{~nm}$ wavelength. (b) SEM image of a 2D dot array pattern with a half-pitch of $17 \mathrm{~nm}$. 


\section{AL NANOPARTICLES}

Here we discuss the basic features of the plasmon resonances of Al nanoparticles and highlight their differences from $\mathrm{Au}$ and Ag nanoparticles. To start with, we consider spherical nanoparticles in a homogeneous environment using Mie's theory, which provides exact analytical solutions for spherical particles ${ }^{8,9}$. Fig. 2(a) shows extinction spectra of Au, Ag and $\mathrm{Al}$ nanoparticles with diameters of $40 \mathrm{~nm}$ in a silica matrix. The plasmon resonances of $\mathrm{Ag}$ and $\mathrm{Au}$ are similar to the spectra reported extensively in literature ${ }^{8,9}$. The sharp extinction peaks in blue and green regions result from the resonant excitation of the dipolar modes of the particle plasmons. The extinction spectrum of the Al nanoshperes shows two distinct resonances in the UV region. The peak at about $250 \mathrm{~nm}$ is due to the dipolar plasmon resonance and the peak at about $190 \mathrm{~nm}$ originates from the quadrupolar mode.

Fig. 2(b) shows extinction spectra of $\mathrm{Al}$ nanospheres with different diameters. With increasing particle size, the peaks exhibit a red-shift and broadening due to retardation effects and higher order plasmon modes becoming more pronounced. Although emergence of higher multipole resonances with increasing particle size may be interesting for some applications, this would imply increasingly stringent restrictions on particle size distribution; for large particles, inhomogeneous broadening as a consequence of finite particle size distribution would smear out all the peaks and make it impossible to observe any resonant features. In the small particle size limit $(d<10 \mathrm{~nm})$, only the dipolar plasmon mode remains and inhomogeneous broadening is not important. However, the peak position is at about $180 \mathrm{~nm}$ (in a silica matrix) and for explicit observation of the plasmon peak, relatively difficult optical experiments are required in the vacuum ultraviolet region. For these reasons the literature on plasmonic properties of $\mathrm{Al}$ is very limited.
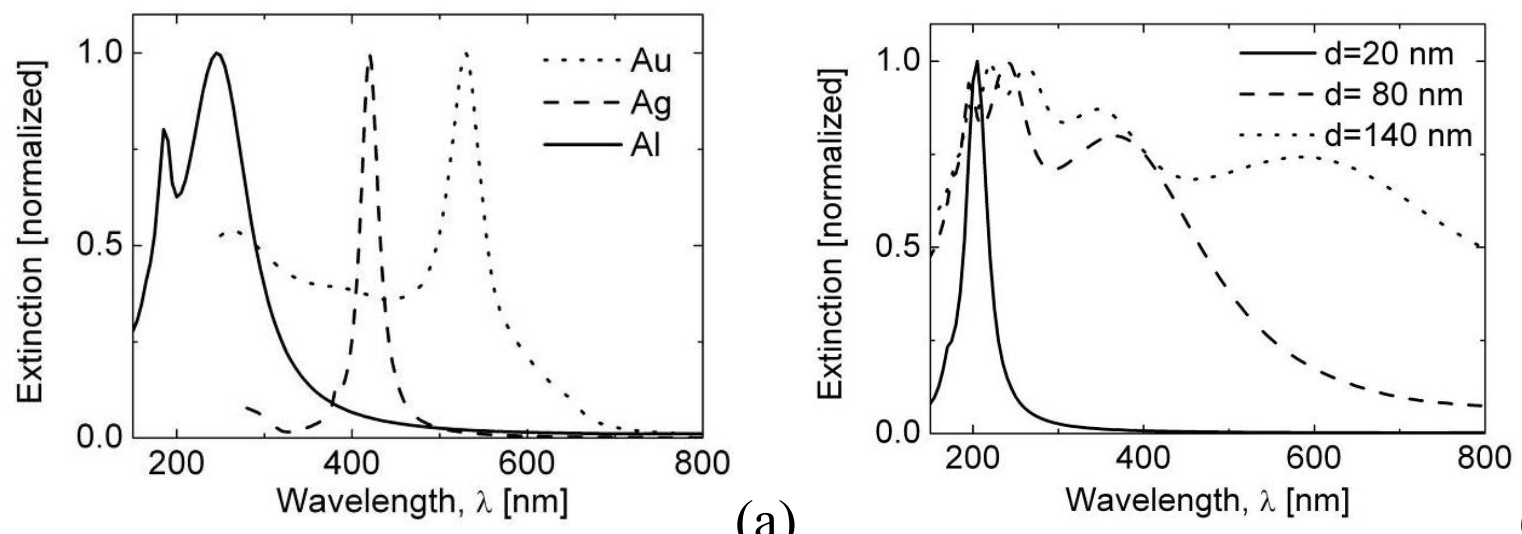

(a)

Figure 2: (a) Calculated extinction spectra of spherical $\mathrm{Au}, \mathrm{Ag}$ and $\mathrm{Al}$ nanoparticles with a diameter of $40 \mathrm{~nm}$ in a silica matrix. (b) Calculated extinction spectra of spherical Al nanoparticles with diameters of $20 \mathrm{~nm}, 80 \mathrm{~nm}$, and $140 \mathrm{~nm}$ in a silica matrix. Calculations are based on Mie's formulae; the optical constants are taken from Ref. [4] and [5].

To obtain ordered and well-defined Al nanoparticles with low size dispersion, we used EUV interference lithography, physical vapor deposition, and lift-off. Sample preparation started with spin-coating of $80 \mathrm{~nm}$-thick polymethylmethacrylate (PMMA) on quartz (HPFS) wafers and with baking at $180^{\circ} \mathrm{C}$ for $3 \mathrm{~min}$. Subsequently, hydrogen silsesquioxane (HSQ) of $30 \mathrm{~nm}$ thickness was spin-coated on the PMMA film and the samples were baked at $90^{\circ} \mathrm{C}$ for 3 min. Following the EUV exposure, HSQ photoresist was developed in a tetra-methyl ammonium hydroxide (TMAH) solution with a normality of 2.6, resulting in hole patterns in HSQ. Reactive ion etching (RIE) etching was used to transfer the pattern into the PMMA layer. This step produces bilayer structures with an undercut that facilitates the 
subsequent lift-off process. Thermal evaporation of aluminum was performed at a base pressure of better than $1 \times 10^{-6}$ mbar with a deposition rate of about $0.5 \mathrm{~nm} / \mathrm{s}$. Finally, the lift-off process was carried out in an acetone bath with ultrasonic agitation.

Fig. 3(a) shows a SEM image of a typical sample. The nanoparticles reveal some roughness due to the finite grain size of Al crystals that are formed within the nanoparticles. From the SEM image we can conclude that the typical grain size is much smaller than the average particle size. The area of the particle arrays was about $1 \mathrm{~mm}^{2}$. Extinction spectra of the nanoparticle arrays were measured using a standard UV/Vis/NIR spectrometer. The measured extinction spectrum of Al nanoparticle array with particle diameter of $40 \mathrm{~nm}$ and lattice constants of $90 \mathrm{~nm}$ and $100 \mathrm{~nm}$ is plotted in Fig. 3(b). The spectrum exhibits a sharp and narrow peak at about $270 \mathrm{~nm}$. Obviously, this resonant peak is the dipolar mode of the surface plasmon resonance of the $\mathrm{Al}$ nanoparticles.
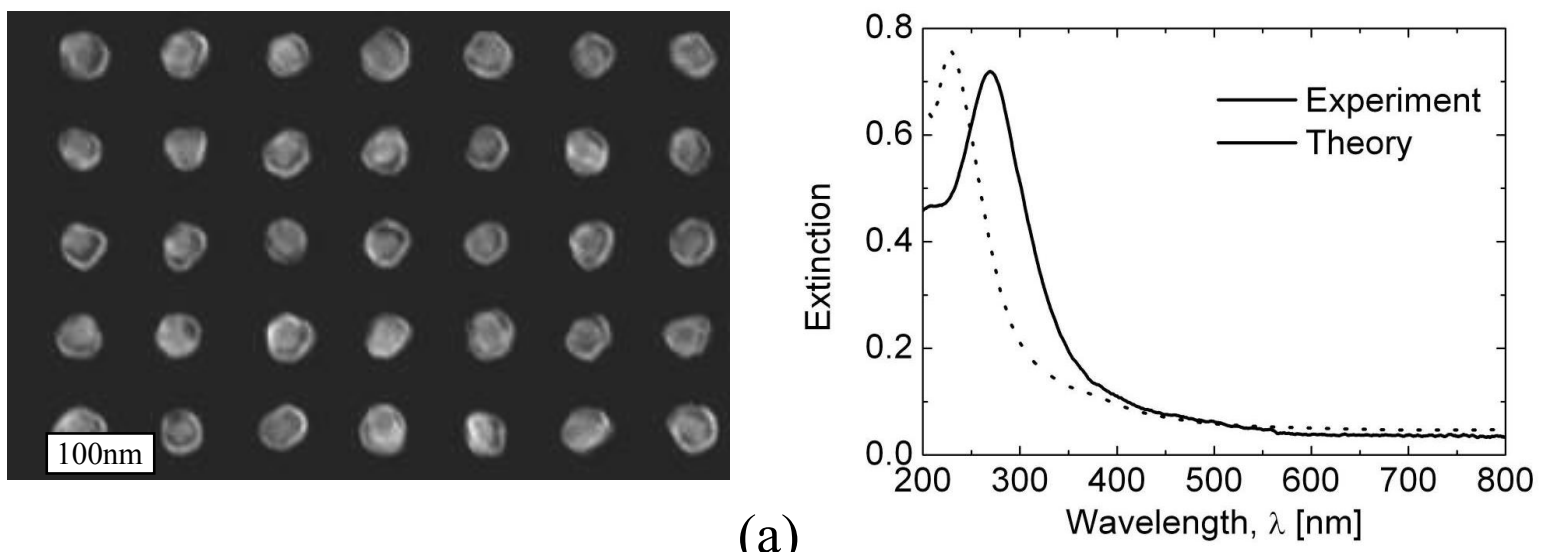

(b)

Figure 3: (a) SEM image of Al nanoparticle arrays with average particle diameter of $40 \mathrm{~nm}$ and thickness of $30 \mathrm{~nm}$. The period of the arrays is $90 \mathrm{~nm}$ and $100 \mathrm{~nm}$ along the principle axes. (b) Experimental and theoretical extinction spectra of Al nanoparticle arrays.

We calculated the extinction spectrum of the structure using a finite-difference time-domain (FDTD) method ${ }^{10,11}$. Al nanoparticles are modeled as cylinders of $40 \mathrm{~nm}$ diameter and $30 \mathrm{~nm}$ height on a semi-infinite substrate; the tabulated values of the complex dielectric constants of $\mathrm{Al}$ were taken from Ref. [5]. The comparison of the experimental and the theoretical spectra in Fig. 3(b) in general reveals good agreement, but there is a discrepancy of $40 \mathrm{~nm}$ in the peak positions. In the FDTD model, the nanoparticles are assumed to be identical and perfect cylinders whereas the real $\mathrm{Al}$ nanoparticles have rather arbitrary shapes due to the finite crystallite size of evaporated aluminum. In addition to this shape dispersion, there is about $5 \%$ size dispersion over the large area of the particle array. The finite size and shape dispersion leads to inhomogeneous broadening in which the experimental peak width is slightly broader than the theoretical peak width. Moreover, in the FDTD model, the natural oxide layer of Al is not taken into account. From optical measurements on $\mathrm{Al}$ surfaces ${ }^{12}$ and TEM analysis of $\mathrm{Al}$ nanoparticles ${ }^{13}$ it is well known that a thin oxide layer of $2-3 \mathrm{~nm}$ is formed on the metal surface upon exposure to air. Modeling of a thin oxide layer in FDTD calculations would have required sub-nanometer grid sizes and therefore unreasonably long computing times. On the other hand, calculations based on dipolar approximation of a coated ellipsoidal nanoparticle ${ }^{8}$ indicate that the existence of the natural oxide layer causes a red-shift of the plasmon resonance wavelength by 5 to $10 \mathrm{~nm}^{14}$. Further detailed analysis ${ }^{14}$ demonstrates that the optical constants reported in literature show a significant variation and that the major source of discrepancy between experiment and theory in Fig. 3(b) arises from differences between the optical constants taken from literature and those of the actual material. Bulk oxidation during the deposition and electron scattering by grain 
boundaries substantially change the optical properties of physically-deposited Al and are highly sensitive to the deposition rate and the base pressure of the deposition chamber ${ }^{15}$.

\section{AL HOLE ARRAYS}

Having seen that Al nanostructures exhibit plasmon resonances in the UV range, we will now present a typical manifestation of plasmon resonances. Since the work of Ebbesen et al. ${ }^{16}$ it has been known that an optically-thick metal film perforated with a periodic array of subwavelength holes transmits light remarkably efficiently at certain resonant wavelengths. Although the underlying mechanisms for the extraordinary transmission are still a matter of debate, it is widely accepted that the resonant excitation of surface plasmon polariton (SPP) Bloch waves supported by the periodic surface structure significantly contributes to the extraordinary transmission ${ }^{1}$. Although the SPP model predicts the positions of some of the observed maxima in the transmission spectra fairly well, the measured spectra often include more features and are influenced by many optical processes and structural parameters. For instance, Wood's anomaly and cavity modes related to grating constant and aperture geometry, respectively, can complicate the spectra and generate additional maxima and minima ${ }^{17}{ }^{18}$. Localized surface plasmons (LSP) can also be excited in the holes, which substantially alter the observed intensities or create new transmission peaks ${ }^{19}$. The phenomenon can be scaled to any frequency in the electromagnetic spectrum by creating hole arrays that have periodicity on the same order as the wavelength, as long as surface plasmon polaritons (SPP) exist or can be mimicked by so-called metamaterials ${ }^{20}$. Enhanced transmission through subwavelength holes has been demonstrated in a large spectral range extending from the visible to the microwave regions ${ }^{1}$. The plasmonic properties of $\mathrm{Al}$ enable us to extend this phenomenon into the UV region.

To obtain free-standing Al films perforated with nanoholes, a $110 \mathrm{~nm}$ thick polymethylmethacrylate (PMMA) film was spin-coated on a $75 \mathrm{~nm}$ thick free-standing $\mathrm{Si}_{3} \mathrm{~N}_{4}$ film. Hole arrays were obtained in the photoresist after exposure with four-beam EUV-IL followed by the development of the PMMA film. Then Al was shadow-evaporated on the sample with a tilt angle of about 50 degrees with respect to the normal while the substrate was rotated around the normal direction. This procedure coats the photoresist film with a uniform layer of metal. Remarkably, the holes remain open despite the considerable thickness of the metal film ( $90 \mathrm{~nm}$ in this example) with respect to the hole size, as seen in Fig. 4(a). Finally, the $\mathrm{Si}_{3} \mathrm{~N}_{4}$ membrane and the PMMA were removed by RIE from the backside, resulting in a free-standing Al nano-mesh.

The transmission spectra were recorded using a commercial spectrometer in the wavelength range of 190-800 nm. The spectra at incidence angles of 0 to 60 degrees with $5^{\circ}$ increments are shown in Fig. 4(b). The maximum absolute transmission achieved at the resonant peak position is $38 \%$. The normalized transmittance of the holes, namely the ratio of the transmitted light to the incident light on the holes, is as large as $130 \%$. With increasing incidence angle, the peaks at about $260 \mathrm{~nm}$ and $350 \mathrm{~nm}$ remain stationary in position, whereas the other strong peak, which is at about $380 \mathrm{~nm}$ at normal incidence, shifts to longer wavelengths. This peak shows a Fano-type resonance, which is more evident at high incidence angles.

We start the analysis of the transmission data by plotting the dispersion behavior of the observed peaks in Fig. 5, where large symbols indicate the strong peaks and small symbols stand for the weaker peaks. Solid lines denote the calculated dispersion curves of the SPPs on a flat Al-air interface based on published values of the optical constants of $\mathrm{Al}^{5}$. We attribute the major peak at $380 \mathrm{~nm}$ wavelength (at normal incidence) to a SPP resonance mode, since it shows a dispersion similar to the curve associated with the reciprocal lattice vector $\boldsymbol{G}(i=-1, j=0)$. As the measured curve approaches $k_{x}=0$ it diverges from the predicted dispersion curve. This is an expected effect, since the periodically structured surface leads to a bandgap at the intersection points of the dispersion curves. The width of the bandgap depends on many factors, such as the shape, the effective area, and the depth of the holes ${ }^{21}$. 
Further, we attribute the two other major peaks $(260 \mathrm{~nm}$ and $350 \mathrm{~nm})$ to localized plasmon resonances ${ }^{22}$. The fact that their positions show no dependence on the angle of incidence, i.e. they are dispersionless, eliminates SPP modes as potential origins for these two peaks. The origin of the peak at $260 \mathrm{~nm}$ is more difficult to ascertain since at this wavelength the first-order diffraction channel is already open and the propagating modes exist. Since SPPs are polarization-dependent, they can be more clearly distinguished with polarized light. The inset in Fig. 4(b) shows the transmission down to $300 \mathrm{~nm}$ wavelength for $s$ - and $p$-polarized light at the incidence angle of $45^{\circ}$. As expected, SPPs are not excited in $s$-polarization and only a broad LSP peak is observed. In contrast, both SPP and LSP peaks are observed in $p$-polarization. Although the peaks attributed to LSPs must be the same for both polarizations as a consequence of the symmetry of the structure, the LSP peak seems to be slightly modified due to the presence of the SPP resonance.

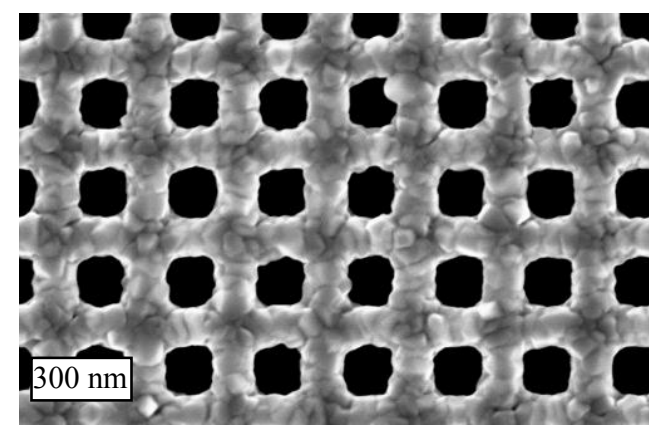

(a)

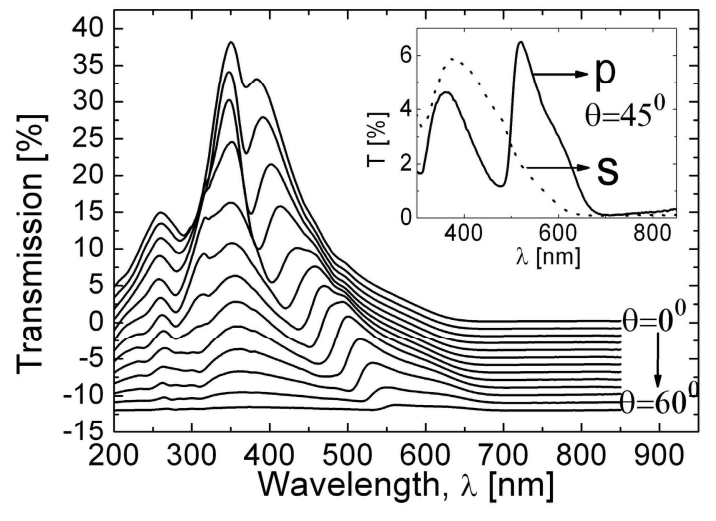

(b)

Figure 4: (a) SEM image of the free-standing Al hole array. The hole period is $283 \mathrm{~nm}$, hole width is $150 \mathrm{~nm}$, and the total area of the hole array is $400 \times 400$ square microns. The Al film thickness is $90 \mathrm{~nm}$. (b) Zeroth-order transmission spectra of the free-standing hole array for different incidence angles using unpolarized light. For clarity, the spectra are plotted with $1 \%$ vertical offset. The inset shows the transmission at $45^{\circ}$ for $s$ - and $p$-polarized light.

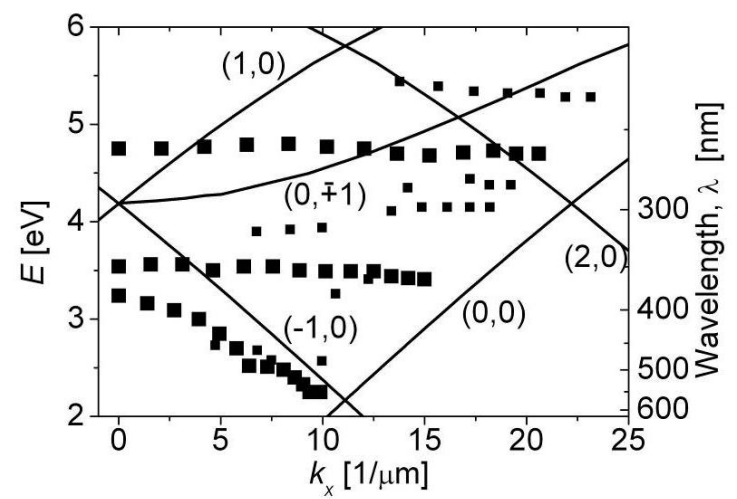

Figure 5: Dispersion curves of the resonant peaks in the transmission spectra of the Al hole array extracted from Fig. 4(b). The large squares denote the larger peaks and small squares indicate the weak peaks in the spectra. The solid lines are the predicted dispersion curves of the surface plasmons of a flat Al-air interface, accompanied by the reciprocal lattice vector with indices $(i, j)$. 


\section{CONCLUSIONS}

In summary, we have demonstrated that $\mathrm{Al}$ nanoparticles and hole arrays show strong and sharp plasmon resonances down to the deep-UV region. Enhanced transmission in the UV range through Al hole arrays is observed, which results from resonance excitation of the SPP modes and localized surface plasmons. We have shown that Al is a good plasmonic material and that its plasmonic properties are very similar to those of "standard" plasmonic materials such as Au and Ag. The major difference of $\mathrm{Al}$ is that its plasmonic resonances are at shorter wavelengths due to the optical properties of aluminum. This makes Al particularly interesting, because there is no other material available which can operate below the wavelength of $300 \mathrm{~nm}$. Extending plasmonics into the UV region will open up new research areas and may enable new applications such as surface enhanced Raman spectroscopy ${ }^{23}$ (SERS) in this spectral region. Localized plasmon sensors $^{3}$, which sense the refractive index changes in the proximity of nanoparticles, can have a higher sensitivity in cases where the refractive index contrast of the analytes is higher in the UV wavelengths. Plasmon waveguides ${ }^{1}$ should have a better lateral confinement by operating at short wavelengths. The exploitation of extraordinary optical transmission of $\mathrm{Al}$ hole arrays could be used in the development of efficient UV filters and beam splitters. Subwavelength photolithography using surface plasmons ${ }^{24,25}$ should achieve better resolution via deep-UV excitation. Another interesting application of plasmonic resonances of $\mathrm{Al}$ nanoparticles might be in energetic nanomaterials ${ }^{26}$. Optical characterization can be used to monitor fundamental processes such as oxidation and rupture of Al nanoparticles.

Part of this work was performed at the Swiss Light Source, Paul Scherrer Institute, Villigen-PSI, Switzerland.

\section{REFERENCES}

1. W. L. Barnes, A. Dereux, and T. W. Ebbesen, "Surface plasmon subwavelength optics," Nature 424, 824 (2003).

2. E. Ozbay, "Plasmonics: Merging photonics and electronics at nanoscale dimensions," Science 311, 189 (2006).

3. E. Hutter and J. H. Fendler, "Exploitation of localized surface plasmon resonance," Adv. Materials 16, 1685(2004).

4. P. B. Johnson and R. W. Christy, "Optical constants of noble metals," Phys. Rev. B 6, 4370 (1972).

5. E. D. Palik, ed., Handbook of Optical Constants of Solids, Academic, 1985.

6. H. H. Solak, C. David, J. Gobrecht, L. Wang, and F. Cerrina, "Multiple-beam interference lithography with electron beam written gratings," J. Vac. Sci. Technol. B 20, 2844-2848 (2002).

7. H. H. Solak, Y. Ekinci, P. Käser and S. Park, "Photon-beam lithography reaches 12.5 nm half-pitch resolution," J.

Vac. Sci. Technol. B 25, 91 (2007).

8. C. F. Bohren and D. R. Huffman, Absorption and Scattering of Light by Small Particles, Wiley, New York, 1983.

9. U. Kreibig and M. Vollmer, Optical Properties of Metal Clusters, Springer Series in Material Science Vol. 25, Springer, Berlin, 1995.

10. A. Taflove and S. C. Hagness, Computational Electrodynamics: The finite-difference time-domain method, $3^{\text {rd }}$ Edition, Artech House, 2005.

11. Y. Ekinci, H. H. Solak, C. David and H. Sigg, "Bilayer Al wire-grids as broadband and high-performance polarizers," Opt. Express 14, 2323 (2006).

12. R. B. Pettit, J. Silcox and R. Vincent, "Measurement of surface-plasmon dispersion in oxidized aluminum films," Phys. Rev. B 11, 3116 (1975).

13. V. Y. Gertsman and Q. S. M. Kwok, "TEM investigation of nanophase aluminum powder," Microsc. Microanal. 11, 410 (2005).

14. Y. Ekinci, H. H. Solak, and J. F. Löffler, “Al Plasmon resonances of aluminum nanoparticles,” to be submitted.

15. M. D. Tillin and J. R. Sambles, "A surface plasmon-polariton study of the dielectric constants of reactive metals: Aluminum," Thin Solid Films 167, 73 (1988).

16. T. W. Ebbesen, H. J. Lezec, H. F. Ghaemi, T. Thio, and P. A. Wolff, "Extraordinary optical transmission through sub-wavelength hole arrays," Nature 391, 667 (1998). 
17. L. Martin-Moreno, F. J. Garcia-Vidal, H. J. Lezec, K. M. Pellerin, T. Thio, J. B. Pendry, and T. W. Ebbesen, “Theory of extraordinary optical transmission through subwavelength hole arrays," Phys. Rev. Lett. 86, 1114 (2001).

18. F. Marquier, J.-J. Greffet, S. Collin, F. Pardo, and J. L. Pelouard, "Resonant transmission through a metallic film due to coupled modes," Opt. Express 13, 70 (2005).

19. Degiron and T. W. Ebbesen, "The role of localized surface plasmon modes in the enhanced transmission of periodic subwavelength apertures," J. Opt. A: Pure Appl. Opt. 7, S90 (2005).

20. A. P. Hibbins, M. J. Lockyear, I. R. Hooper, and J. R. Sambles, "Waveguide arrays as plasmonics metamaterials: Transmission below cutoff," Phys. Rev. Lett. 96, 073904 (2006).

21. A. Degiron, H. J. Lezec, W. L. Barnes, and T. W. Ebbesen, "Effects of hole depth on enhanced light transmission through subwavelength hole arrays," Appl. Phys. Lett. 81, 4327 (2002).

22. Y. Ekinci, H. H. Solak and C. David, "Extraordinary optical transmission in the ultraviolet region through aluminum hole arrays," Opt. Lett. 32, 172 (2007).

23. K. A. Willets and R. P. Van Duyne, "Localized surface plasmon resonance spectroscopy and sensing," Annu. Rev. Phys. Chem. 58, 267 (2007).

24. W. Srituravanich, N. Fang, C. Sun, Q. Luo, and X. Zhang, "Plasmonic nanolithography," Nano Lett. 4, 1085 (2004). 25. D. B. Shao and S. C. Chen, "Surface-plasmon-assisted nanoscale photolithography by polarized light," Appl. Phys. Lett. 86, 253107 (2005).

26. D. D. Dlott, “Thinking big (and small) about energetic materials," Mater. Sci. Technol. 22, 463 (2006). 